\title{
Influencia de la geometría del proyectil en ensayos dinámicos de tracción
}

\author{
Influence of the projectile's geometry on \\ dynamic tension experiment
}

\author{
Roberto Alonso González Lezcano ${ }^{1}$, Eduardo Jose López Fernández ${ }^{1}$, \\ Sonia Cesteros García ${ }^{1}$, Gastón Sanglier Contreras ${ }^{1}$
}

\footnotetext{
${ }^{1}$ Departamento de Arquitectura y Diseño. Escuela Politécnica Superior, Universidad CEU, San Pablo, Madrid, España. e-mail: rgonzalezcano@ceu.es, eduardojose.lopezfernandez@ceu.es, son.cesteros.ce@ceindo.ceu.es, sanglier.eps@ceu.es
}

\begin{abstract}
RESUMEN
El enfoque principal de este estudio es discutir la influencia de la longitud del proyectil en los resultados de un experimento de la barra Hopkinson de tracción (SHTB). Se han realizado simulaciones por medio de elementos finitos utilizando el software comercial ABAQUS en ensayos de tracción a altas velocidades de deformación en muestras de aleación de aluminio 7017T73 variando la longitud del proyectil. Los análisis por elementos finitos se han aplicado para simular los efectos de la variación de la longitud del proyectil en las mediciones obtenidas en las barras incidente, reflejada y transmitida. Se han obtenido diferentes velocidades de deformación, al variar la longitud del proyectil permaneciendo su velocidad constante. Los resultados de la simulación muestran que la longitud del proyectil tiene un efecto significativo en la tensión obtenida en la muestra y también en la curva de tensión-deformación. Se puede concluir que la longitud del proyectil es un factor que influye en los ensayos de tracción dinámica, ya que tiene un efecto significativo en la tensión obtenida dentro de la probeta. Las simulaciones también proporcionan información complementaria a los experimentos y una comprensión profunda del comportamiento de la probeta.
\end{abstract}

Palabras clave: Ensayos dinámicos de tracción, caracterización mecánica, altas velocidades de deformación, simulaciones numéricas, barra Hopkinson de tracción, mecánica experimental, ensayo de materiales.

\begin{abstract}
The focus of this study is to discuss the influence of the projectile's length on the results of an experiment of the Hopkinson Traction Bar (SHTB). Simulations have been made by means of finite elements using the commercial software ABAQUS in tests of traction at high speeds of deformation in samples of aluminum alloy 7017T73 varying the length of the projectile. Finite element analyses have been applied to simulate the effects of the variation in the length of the projectile in the measurements obtained in the incident, reflected and transmitted rods. Different warp speeds have been obtained by varying the length of the projectile while remaining constant speed. The results of the simulation show that the length of the projectile has a significant effect on the tension obtained in the sample and on the curve of tension-deformation. It can be concluded that the length of the projectile is a factor influencing the tests of dynamic traction, since it has a significant effect on the tension obtained inside the specimen. The simulations also provide complementary information to the experiments and a thorough understanding of the specimen's behavior.
\end{abstract}

Keywords: dynamic tension experiment, mechanical characterization, high-strain-rate testing, numerical simulations, split Hopkinson tension bar, experimental mechanics, material testing.

\section{INTRODUCCIÓN}

El conocimiento sobre el comportamiento mecánico de los materiales es fundamental para optimizar su uso en diferentes diseños. Una parte fundamental en el comportamiento mecánico de un material es el estudio dinámico del mismo. Para estudiar el comportamiento dinámico de un material se utilizan diferentes métodos entre los cuales, se encuentra el ensayo de barra Hopkinson [1][26].

Mediante este tipo de ensayo, se pueden estudiar materiales a altas velocidades de deformación (200 - 
$104 \mathrm{~s}^{-1}$ ) [2]; tanto en los modos de tracción como de compresión.

Algunos ejemplos de aplicaciones en las que es necesario el conocimiento del comportamiento de los materiales a altas velocidades son:

- En el campo aeronáutico, donde los elementos estructurales podrían sufrir impactos durante el vuelo, como podría ser la basura espacial sobre satélites, el impacto de aves en las zonas cercanas a aeropuertos, etc.

- En el campo de los transportes, en el que aparecen condiciones de trabajo violentas que tienen que ser estudiadas para impedir cualquier anomalía por parte de los materiales utilizados.

- En el campo balístico donde se trabaja con impactos a altas velocidades, blindajes, etc.

Todos los campos mencionados anteriormente serán ejemplos de la utilidad de este tipo de estudios, donde se mostrarán una diferencia entre el comportamiento cuasi-estático y dinámico en los materiales [2].

Así como es relativamente sencillo detener un ensayo cuasi-estático en un nivel de deformación determinado, y de esta manera, poder estudiar microscópicamente el comportamiento del material, presenta una mayor dificultad el detener un ensayo dinámico a altas velocidades de deformación [3] [4].

Por tanto, este estudio irá enfocado al análisis del comportamiento de materiales sometidos a altas velocidades de deformación, desarrollando una herramienta de trabajo que permita someter a los materiales a distintos niveles y velocidades de deformación de forma controlada [5].

Los ensayos de impacto o ensayo de resiliencia a medias o altas velocidades de deformación sirven para determinar la fragilidad o resistencia que opone un material a la rotura. Esta resistencia se conoce por el nombre de resiliencia y se expresa en Julios. Para realizar el ensayo se emplea el péndulo de caída o Péndulo Charpy que mide la energía residual existente después de la rotura de la muestra de material.

Hoy en día se conocen varias técnicas experimentales para realizar estudios sobre el comportamiento dinámico de los materiales. En función de las velocidades de deformación a las que se someten los materiales ensayados, existen los siguientes modelos experimentales:

$$
\begin{aligned}
& \text { - } \quad \text { El péndulo Charpy y torre de caída (hasta } 500 \mathrm{~s}^{-1} \text { ) } \\
& \text { - } \quad \text { La barra Hopkinson y el cilindro Taylor (entre } 10^{2} \text { y } 10^{4} \mathrm{~s}^{-1} \text { ) } \\
& \text { - } \quad \text { El Cañón de impacto y anillo expandible. }
\end{aligned}
$$

La velocidad de deformación es un factor que puede modificar el comportamiento de un material ante una solicitación mecánica. En muchas aplicaciones los materiales trabajan simultáneamente en condiciones de alta o baja velocidad de deformación, por lo que resulta necesario disponer de técnicas experimentales de caracterización mecánica que permitan reducir estas circunstancias [6].

Para la caracterización de materiales de tracción a alta velocidad de deformación, existen diferentes procedimientos de los cuales cabe destacar el que se describe en este artículo consistente en ejercer un pulso de tracción sobre una probeta y medir en la misma, la deformación y la tensión como funciones del tiempo, Este tipo de ensayo posibilita la obtención de curvas tensión-deformación, bajo condiciones controladas, a fin de obtener propiedades de los materiales cuando son sometidos a altas velocidades de deformación.

Para cumplir con los objetivos de este trabajo, se ha realizado el siguiente planteamiento referentes a la viabilidad del modelo de barra Hopkinson:

1. Simulación numérica de la barra Hopkinson a diferentes velocidades de deformaciones y diferentes proyectiles, empleando el método de los elementos finitos.

2. Comparación de las tensiones y deformaciones del diseño a una misma velocidad de deformación, y estudio del efecto de la geometría del proyectil por medio de simulaciones numéricas.

Se ha utilizado como material de ensayo una aleación de aluminio Al7017T73 como material de ensayo y se ha realizado un modelo de barra Hopkinson que permitiera modificar el efecto del tamaño del proyectil. [7].

\section{MATERIALES Y MÉTODOS}

Para los ensayos se ha empleado una barra Hopkinson, la cual se muestra en la Figura 1, formada por dos barras cilíndricas (incidente y transmisora) y una probeta situada entre ambas barras, que será del material a ensayar. Las barras serán cilíndricas y de diferentes longitudes. El hecho de que sean cilíndricas facilitará el estudio de ondas buscando que sus dimensiones sean las adecuadas para optimizar los ensayos [8]. 


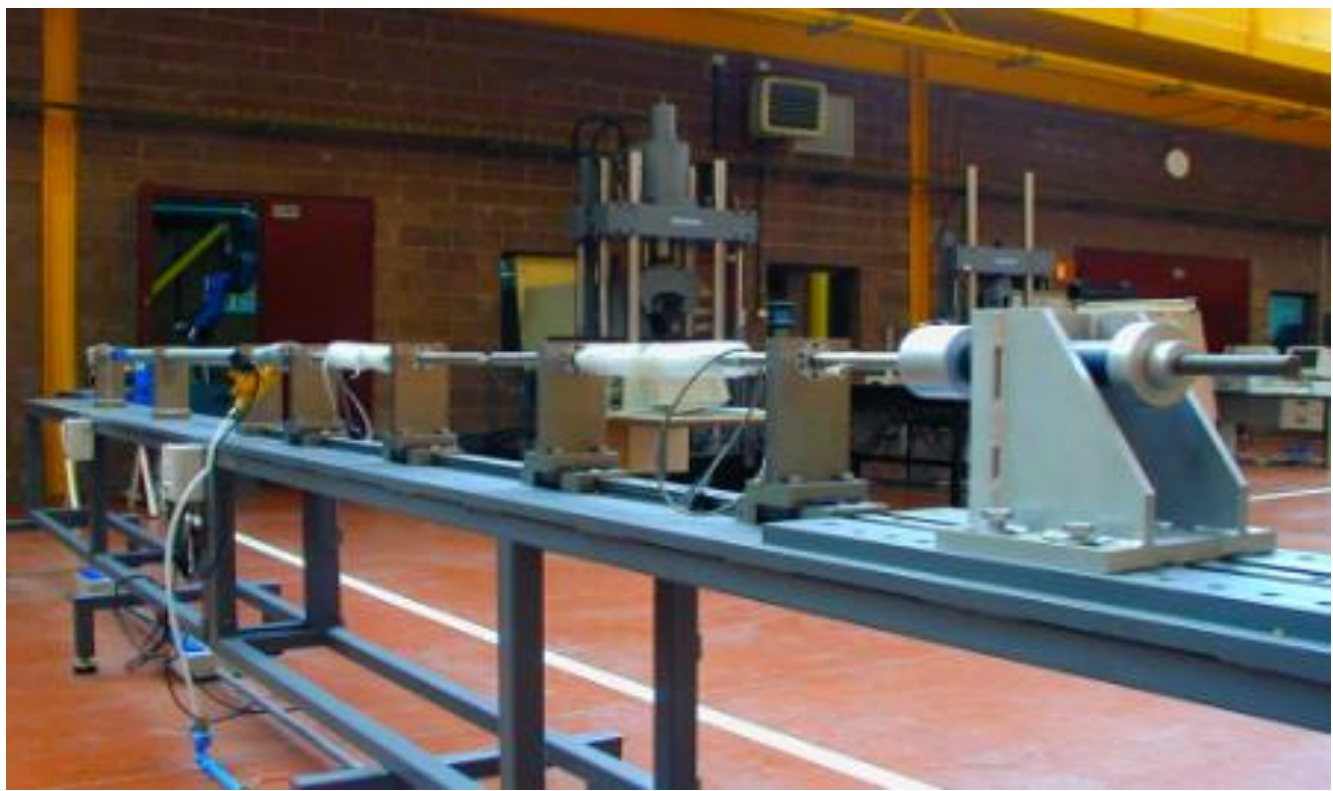

Figura 1: Barra Hopkinson de tracción de la Universidad Carlos III de Madrid.

En cuanto a las propiedades de las barras, tendrán un límite elástico suficientemente elevado, de manera, que al ensayo las afecte lo menos posible [9]. Será fundamental un conocimiento preciso de sus propiedades, ya que de esta precisión dependerá la fiabilidad de los resultados [10].

Las barras irán apoyadas en unos soportes móviles, que a su vez se podrán ajustar sobre guías fijadas a una bancada, de forma, que se asegure el perfecto alineamiento de todos los componentes.

La instrumentación utilizada medirá la deformación que se produce en ambas barras que se hará por medio de dos galgas extensométricas colocadas una en cada barra, como se puede ver en le Figura 2, de manera, que las ondas se recojan con la mayor claridad posible [11]. La información captada por las galgas se enviará a un acondicionador de señal, y de ahí a un osciloscopio, donde se podrá trabajar con los datos del ensayo.

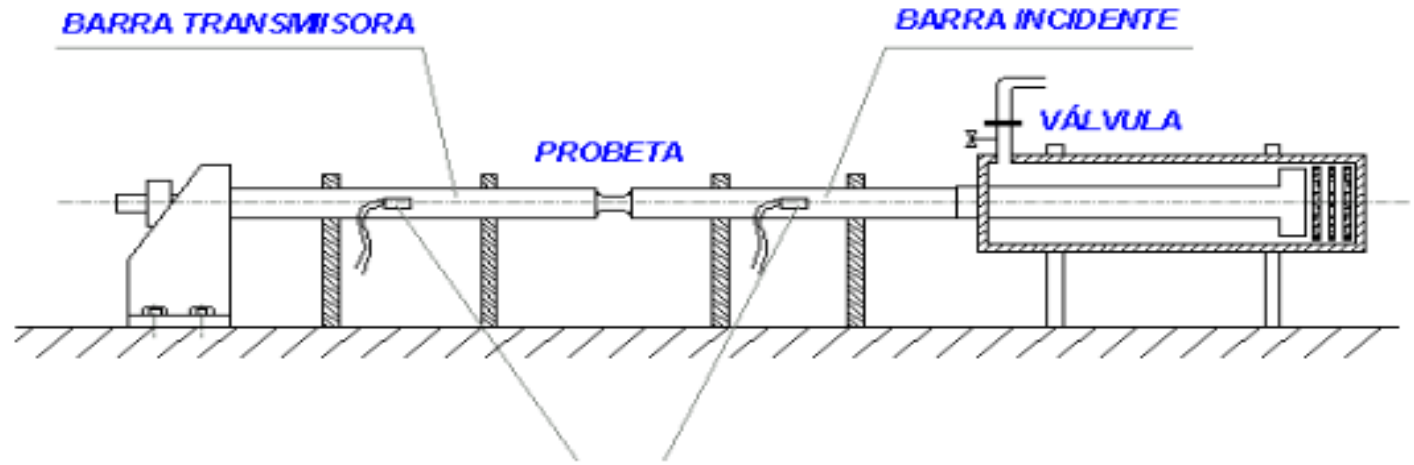

BANDAS EXTENSOMÉTRICAS

Figura 2: Detalle de la colocación de galgas extensométricas en la barra Hopkinson de tracción.

En el ensayo de tracción, la probeta se encuentra roscada en sus extremos a la barra incidente y transmisora. La barra incidente tendrá mayor longitud que la transmisora y es la que recibe el impacto del proyectil. [12].

Siguiendo las recomendaciones de la ASTM E-8 (1994) así como las recomendaciones de Rodríguez (1993) en cuanto a la relación de la longitud del fuste y al diámetro de las probetas, se han utilizado probetas, como la que se muestran en la Figura 3 cuyas dimensiones son: 


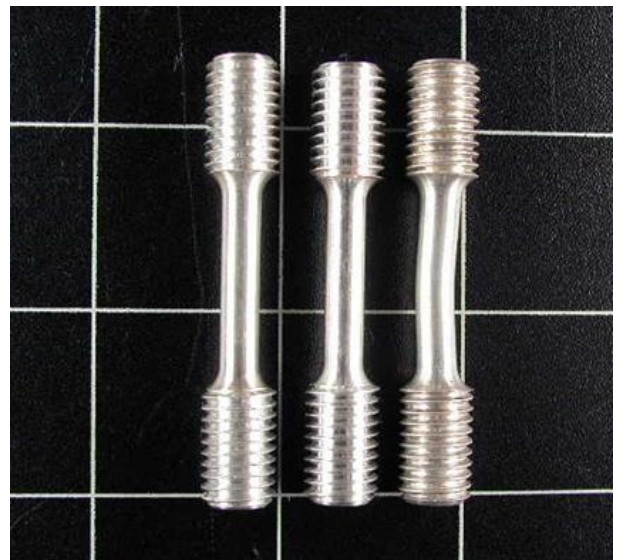

Figura 3: Probetas utilizadas en los ensayos dinámicos.

Longitud del fuste cilíndrico $(\mathrm{L})=25.4 \mathrm{~mm}$

Diámetro fuste $(\mathrm{D})=6.35 \mathrm{~mm}$

Diámetro zona roscada $\left(\mathrm{D}_{\mathrm{r}}\right)=12 \mathrm{~mm}$

Longitud zona roscada $\left(\mathrm{L}_{\mathrm{r}}\right)=14 \mathrm{~mm}$

En la Figura 4 se muestra el diagrama de Lagrange con la representación de ondas elásticas de tensión en barras de un modo sencillo, mediante diagramas espacio- tiempo. Con el apoyo de la información brindada por el Diagrama de Lagrange es posible ubicar la posición de las galgas extensométricas de manera que no exista una superposición de ondas que afecte las lecturas de las mismas.

Una vez obtenidas las deformaciones que dan las galgas extensométricas, se procede a la obtención de la curva tensión-deformación, que es el principal objetivo del ensayo; ara ello, se hace uso de la teoría de ondas unidimensionales.

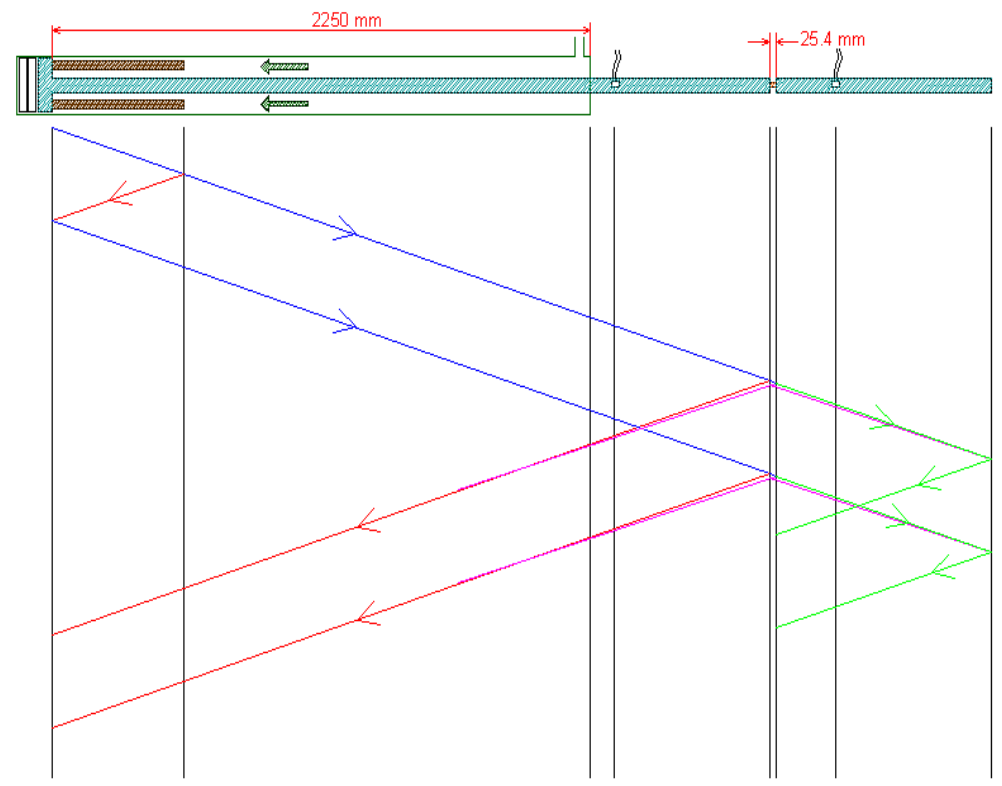

Figura 4: Diagrama de Lagrange para la barra Hopkinson de tracción.

El impacto se realiza con un proyectil, que es del mismo material que las barras. Éste tiene una forma de cilindro hueco y se mueve sobre la barra incidente hasta un tope situado en el extremo de ésta, donde se produce el impacto. [13] [14]. La forma de acelerar el proyectil se realiza mediante un sistema de aire comprimido que tiene una presión máxima de 8 bares [15] [16]. Por tanto, el sistema de ajuste del impacto será una válvula de regulación, la cual, se ajustará a la presión que nos proporcione el impacto requerido [17]. 


\section{ANÁLISIS Y RESULTADOS}

El modelo (Figura 5) se ha realizado utilizando el Software comercial ABAQUS con el cual se pueden realizar muchas cosas: darle al modelo todo tipo de propiedades, hacer uniones e interacciones entre piezas, aplicarle cargas y restricciones, hacer el mallado de las piezas, elegir las variables de salida y ejecutar diferentes casos mediante elementos rectangulares axisimétricos (CAX4R), y hacer un mallado de tipo estructurado.

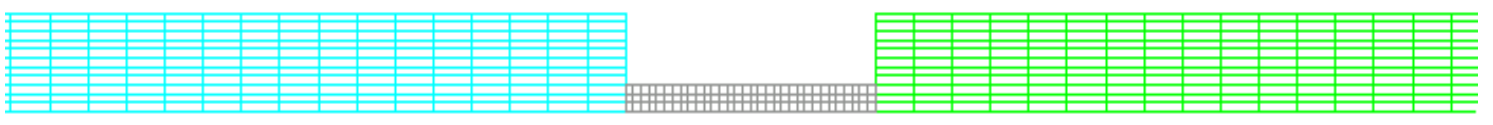

Figura 5: Mallado en barra simple.

Para este estudio, el modelo numérico de barra Hopkinson simple constará de:

- $\quad$ Número de nodos: 10.596

- Número de elementos: 9.678

Los estudios teóricos indican que para las frecuencias que se encuentran en los ensayos en barra Hopkinson, las diferencias entre los elementos interiores y exteriores deberían ser despreciables; por tanto, deberían serlo también en el modelo. [18] [19].

Para comprobarlo, se han elegido varios elementos de la barra incidente y transmisora. Los elementos están situados a la altura de las galgas de cada barra y la distancia respecto al centro de la barra son: 4, 7.5 y $11 \mathrm{~mm}$ (superficie). También se ha tomado la deformación en el centro de las barras.

En los gráficos de las figuras 6 y 7 se puede apreciar que no hay diferencias significativas entre los elementos de las gráficas.

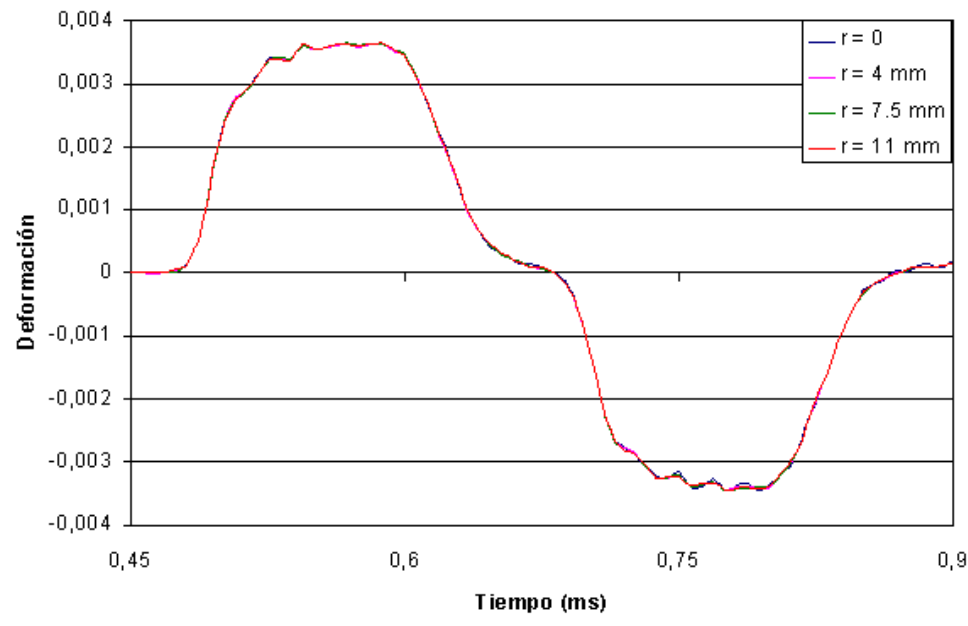

Figura 6: Deformaciones en la barra incidente.

Se realizó un ensayo real en barra Hopkinson y se compararon las ondas incidente y reflejada que indicaban las galgas extensométricas con las ondas incidente y reflejada de un elemento del modelo en la ubicación exacta de las galgas. Para comparar estos resultados se utilizó un proyectil de $33 \mathrm{~cm}$.

El modelo numérico permitió comprobar el cumplimiento de las hipótesis de la teoría de las ondas unidimensionales referente al comportamiento de las barras [20] [21], como lo es el hecho que la deformación en el interior de las barras es la misma que en la superficie [22]. 


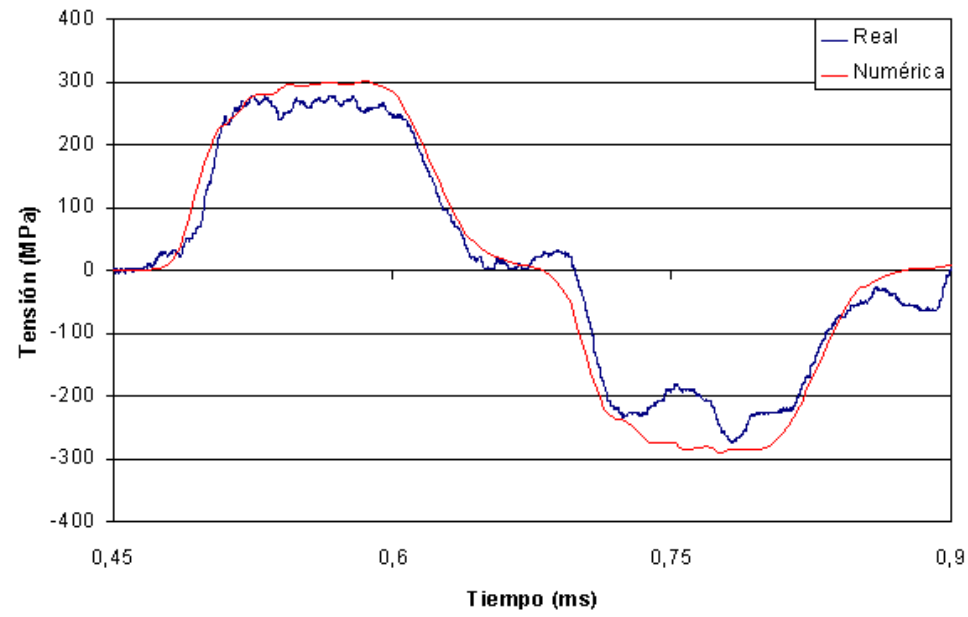

Figura 7: Ondas incidente y reflejada para un ensayo real y numérico.

En la figura 7 se puede apreciar que se aproximan bastante el pulso de la simulación numérica y el pulso del ensayo real. El momento en el cual los pulsos incidente y reflejado comienzan, en ambos casos, es prácticamente el mismo, lo que indica que la velocidad de propagación en el caso real y simulado es la misma.

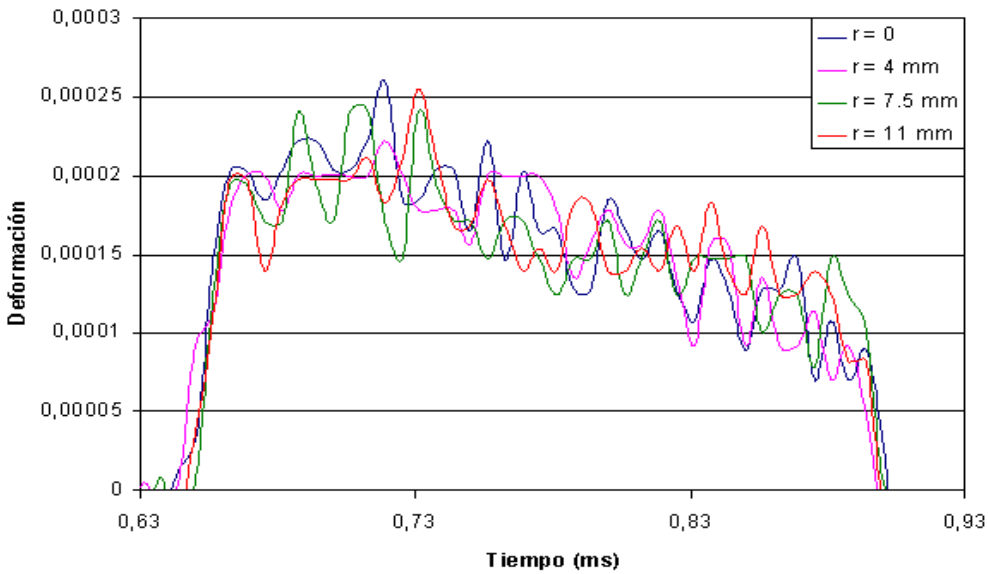

Figura 8: Deformaciones en la barra transmisora.

A partir de la validación del modelo con roscas demostrando que se cumple la teoría de ondas unidimensionales para una velocidad de deformación de $1300 \mathrm{~s}^{-1}$ y una longitud de proyectil de $33 \mathrm{~cm}$, se realizan otras simulaciones numéricas para tensión, deformación (Figura 8) y velocidad de deformación que corresponden a proyectiles de $20 \mathrm{~cm}, 40 \mathrm{~cm}$ y $55 \mathrm{~cm}$.

La figura 9 muestra la influencia de las diferentes longitudes de proyectiles en el comportamiento de las ondas incidentes y reflejadas. 


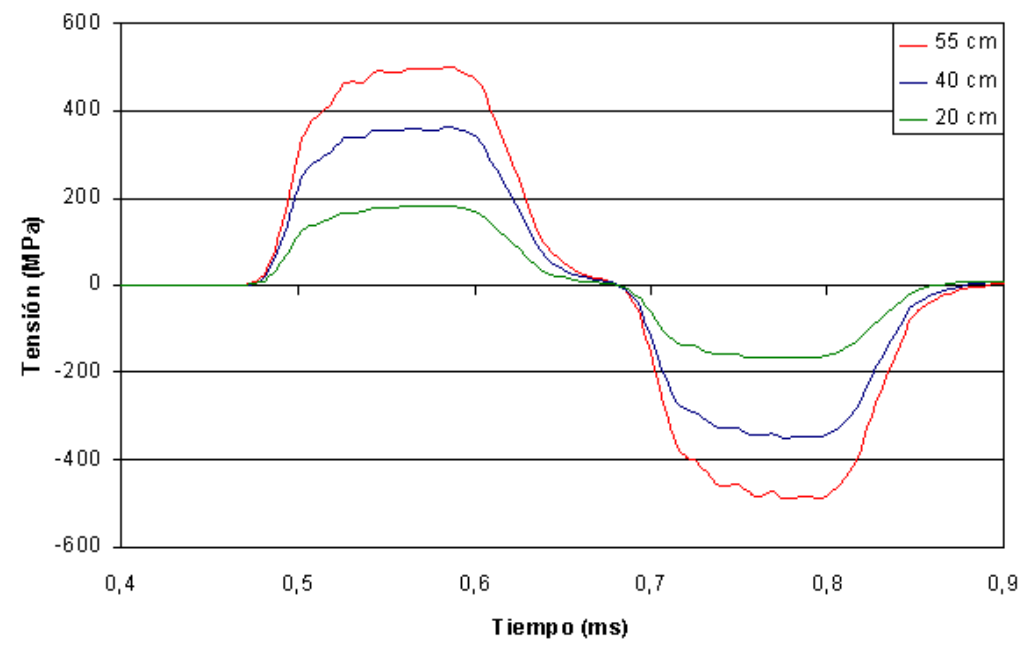

Figura 9: Ondas incidentes y reflejadas de tensión para varias longitudes de proyectil.

Se puede comprobar que el proyectil de mayor longitud $(55 \mathrm{~cm})$ produce una onda de mayor tensión, y el de menor longitud $(20 \mathrm{~cm})$ de menor tensión. El proyectil de $55 \mathrm{~cm}$ produce una onda incidente de 480 MPa de tensión máxima y una onda reflejada de $455 \mathrm{MPa}$. El proyectil de $40 \mathrm{~cm}$ alcanza los $350 \mathrm{MPa}$ en la onda incidente y los $330 \mathrm{MPa}$ en la onda reflejada. El de menor longitud de $20 \mathrm{~cm}$, llega hasta $175 \mathrm{MPa}$ en la onda incidente y en la onda reflejada a $155 \mathrm{MPa}$. Como se observa de los datos obtenidos, la onda incidente es mayor que la reflejada, ya que la onda incidente choca con la probeta, parte de esa tensión se refleja, y parte se transmite a la probeta.

Todas las ondas comienzan en $0,47 \mathrm{~ms}$, y comparando el tiempo que dura cada onda, se observa una pequeña diferencia entre las ondas de los distintos proyectiles. El de mayor longitud, tiene una onda de mayor tiempo. La diferencia es mínima, pues la velocidad con la que viajan las ondas es muy elevada (próxima a $\operatorname{los} 5170 \mathrm{~m} / \mathrm{s}$ ).

Otra diferencia significativa se aprecia en el cambio de incidente a reflejada, se observa como la onda del proyectil menor tiende a ser horizontal; mientras que las ondas de los proyectiles mayores están siempre inclinadas en ese tramo, siendo la onda producida por el de mayor longitud la más inclinada. Esto demuestra que la duración de la onda es mayor cuanto mayor es la longitud del proyectil, y por ello, el cambio de una onda a otra es más rápido. [23] [24]. Las ondas transmitidas se toman de la barra transmisora del modelo. Al igual que en el caso anterior, las medidas se toman en el lugar donde estarían las galgas colocadas en un ensayo real [25]. En la figura 8, se muestra cómo influye la longitud del proyectil en el comportamiento de las ondas transmitidas.

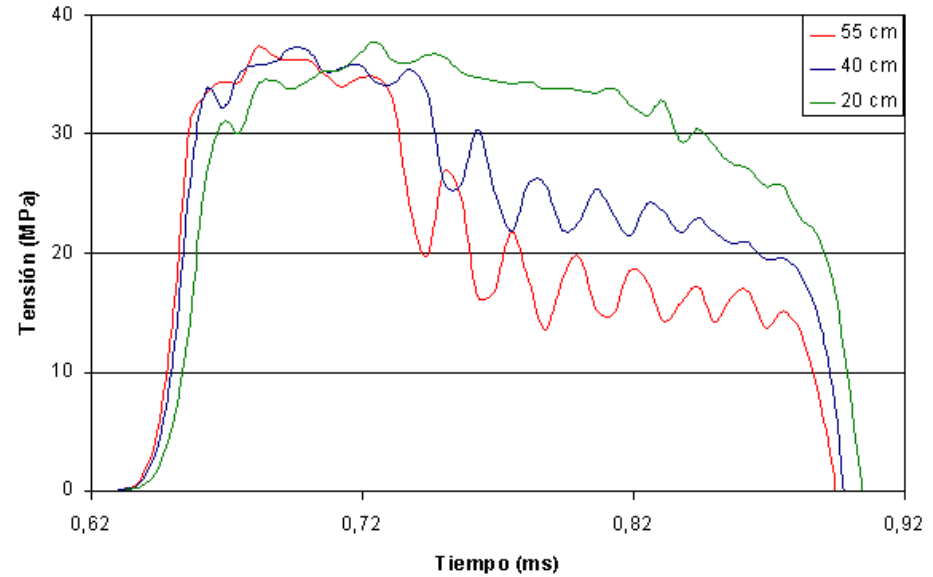

Figura 10: Ondas transmitidas de tensión de los diferentes proyectiles. 
En la figura 10, se observa que las ondas transmitidas de los proyectiles de $55 \mathrm{~cm}$ y $40 \mathrm{~cm}$ tienen un valor máximo muy parecido. La igualdad es debido a las deformaciones alcanzadas por la probeta, la probeta se habría roto en ambos casos. Después de someter a la probeta al impacto del proyectil de $20 \mathrm{~cm}$, la probeta sufre deformaciones menores, no llegando a formar el cuello de estricción. Sin embargo, cuando se analizan los otros dos casos, las probetas sufren deformaciones llegando a formar el cuello de estricción.

La siguiente figura 11 muestra las deformaciones producidas en medio del fuste de la probeta para los diferentes tamaños de proyectil. Se puede observar en la gráfica, que se obtiene mayores deformaciones con el proyectil mayor. Las pendientes de las rectas indican las velocidades de deformación. De aquí se puede deducir que el proyectil mayor tiene una velocidad de deformación más grande que los demás proyectiles pues su pendiente es más inclinada que la del resto de curvas.

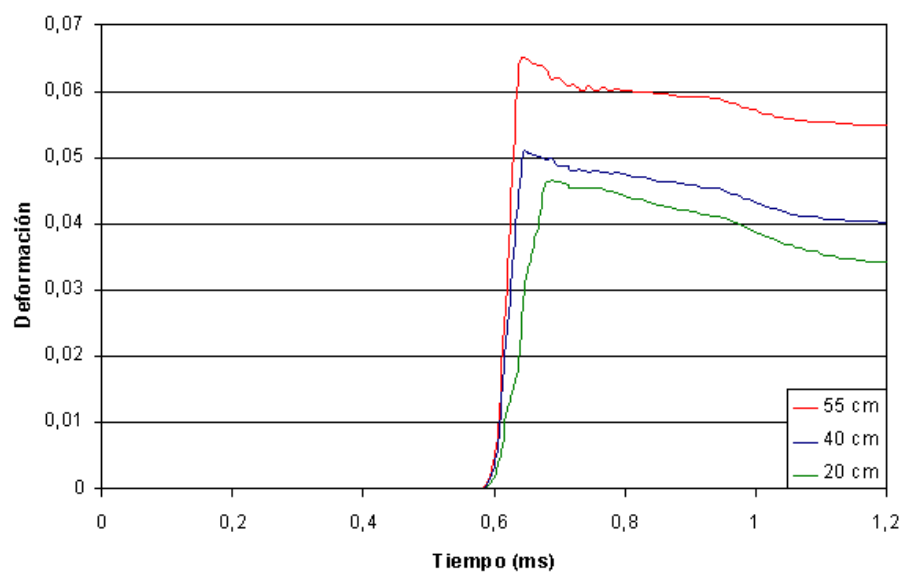

Figura 11: Curva Deformación-tiempo en la probeta.

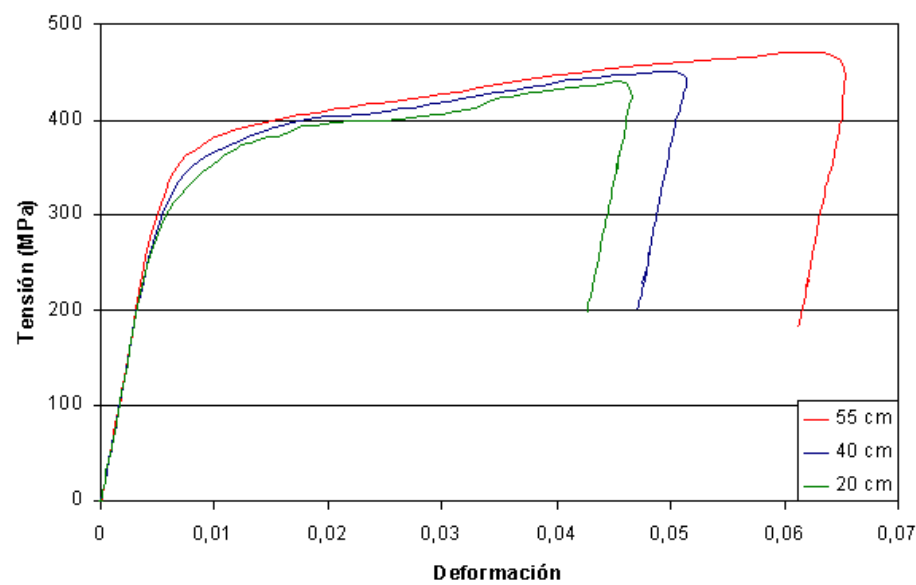

Figura 12: Curva tensión-deformación en la probeta.

La velocidad de deformación en la probeta se ha calculado como la derivada de la curva deformacióntiempo en el punto medio de la muestra. La figura 13, muestra las distintas curvas de velocidad de deformación obtenidas por el cambio en la longitud del proyectil. 


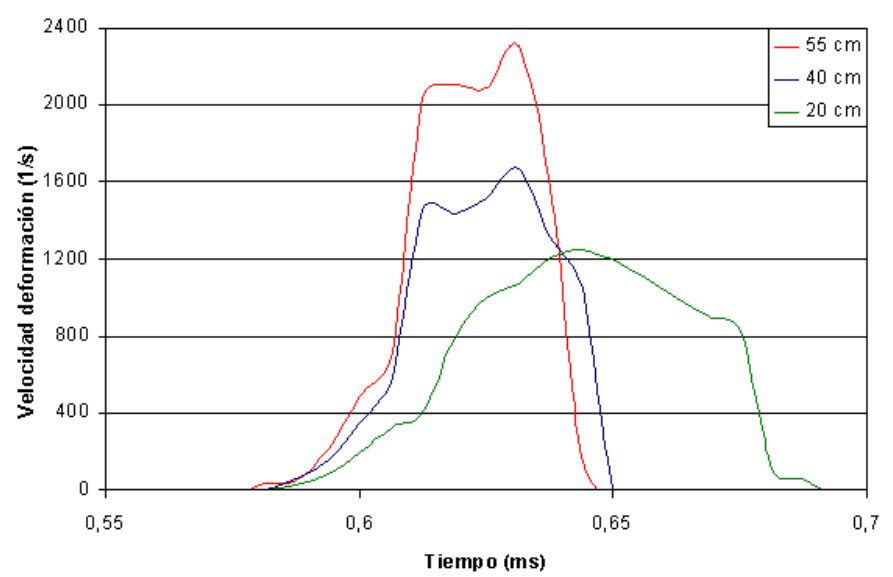

Figura 13: Velocidad de deformación para diferentes tamaños de proyectil.

Como se observa, el proyectil de mayor longitud llega a una velocidad de deformación de $2100 \mathrm{~s}^{-1}$ aproximadamente, mientras que el proyectil de $40 \mathrm{~cm}$ tiene un máximo aproximado de $1500 \mathrm{~s}^{-1}$ y el proyectil menor alrededor de $1000 \mathrm{~s}^{-1}$.

\section{CONCLUSIONES}

Se ha simulado numéricamente la barra Hopkinson de tracción y se han obtenido diferentes velocidades de deformación empleando distintas presiones de impacto, debido al efecto de diferentes longitudes de proyectiles y manteniendo una velocidad constante del proyectil en todos los casos ejecutados.

Se ha observado que el cuello de estricción se forma en la parte del fuste que está más próxima a la barra incidente, puesto que las tensiones máximas se concentran en esa parte, por ello esa parte sufre deformaciones máximas.

Se aprecia que el tiempo que dura el pulso del proyectil de $20 \mathrm{~cm}$ es mayor que el de los demás. Cuanto mayor es la velocidad de deformación del impacto, menor es el tiempo que dura ésta.

Se obtienen mayores deformaciones y mayores velocidades de deformación con el proyectil de mayor longitud, por lo que, si se quisieran ensayar probetas de mayor resistencia a la tracción, se debería aumentar la presión de impacto en la barra incidente, o disponer de proyectiles de mayor longitud.

De cara a trabajos futuros, se consideraría muy útil, poder simular un modelo tridimensional de la barra Hopkinson de tracción donde pudieran ser estudiados los casos de desalineación, concentricidad de los ejes de las barras y la posición de los apoyos, puesto que los elementos axisimétricos están limitados [26].

De sumo interés sería el poder simular casos donde se tengan en consideración el efecto de la temperatura, y medir experimentalmente dicho efecto en los ensayos, ya que, durante la deformación plástica de la probeta se consume energía, y ésta se disipa térmicamente aumentando la temperatura de las zonas de máxima deformación. El material de la probeta con la que se suele trabajar es dúctil, y debido al aumento de temperatura por la deformación, el material se reblandece.

\section{AGRADECIMIENTOS}

Los autores agradecen a la Universidad CEU San Pablo por los recursos e infraestructuras proporcionados. A los autores les gustaría expresar su gratitud al laboratorio de mecánica de medios continuos y teoría de estructuras de la Universidad Carlos III de Madrid.

\section{BIBLIOGRAFÍA}

[1] HARDING, J., WELSH, L., “A tensile testing technique for fiber-reinforced composites at impact rates of strain”, J Mater Sci, v.18, n.6, pp.1810-1826, 1983.

[2] LINDHOLM, U., YEAKLEY L. High strain-rate testing - tension and compression. v.8, n.1, pp. 1-9, 1968.

[3] ALBERTINI, C., MONTAGNANI, M. "Wave-propagation effects in dynamic loading", Nucl Eng Des., v. 37, n.1, pp. 115-124, 1976. 
[4] NICHOLAS, T. "Tensile testing of materials at high-rates of strain”, Experimental Mechanics, v.21, n.5, pp.177-185, 1981.

[5] THAKUR, A, NEMAT-NASSER, S., VECCHIO, K. "Dynamic Bauschinger effect”, Acta Materialia, v.44, n.7, pp. 2797-2807, 1996.

[6] VERLEYSEN, P., DEGRIECK, J. "Experimental investigation of the deformation of Hopkinson bar specimens”, Int J Impact Eng., v. 30, n.3, pp. 239-253, 2004.

[7] GRAY, GT. Classic split-Hopkinson pressure bar testing. Materials Park, OH, ASM International, pp.462-476, 2000.

[8] GAMA, B.A., LOPATNIKOV, S.L., GILLESPIE, J.W. "Hopkinson bar experimental technique: a critical review", Appl. Mech. Rev, v. 57, n.4, pp. 223-250, 2004.

[9] GERLACH, R, KETTENBEIL, C., PETRINIC, N. “A new split Hopkinson tensile bar design”, Int J Impact Eng., v.50, pp. 63-67, 2012.

[10] KOLSKY, H. "An investigation of the mechanical properties of materials at very high rates of loading", Proc Phys Soc B., v. 62, n.11, pp.676, 1949.

[11] VAN SLYCKEN J. “Advanced use of a split Hopkinson bar setup application to TRIP steels”, D.Sc. Thesis, Ghent University, Belgium, 2008.

[12] HAMOUDA, A., HASHMI, M. "Testing of composite materials at high rates of strain: advances and challenges", J Mater Process Techno, v. 77, n.1-3, pp.327-336, 1998.

[13] KHLIF, M, MASMOUDI, N., BRADAI, C. "Polypropylene tensile test under quasi-static and dynamic loading", In: Materials science and technology conference and exhibition (MS\&T), 2012.

[14] VERLEYSEN, P, BENEDICT, V, VERSTRAETE, T, et al., "Numerical study of the influence of the specimen geometry on Split Hopkinson bar tensile test results”, Lat Am J Solids Struct., v.6, n.3, pp.285-298, 2009.

[15] KAISER, M.A. "Advancements in the split Hopkinson bar test”, M.Sc., Virginia Polytechnic Institute and State University, Blacksburg, VA, 1998.

[16] SWANTEK, S, WICKS, A.L., WILSON, L. "An optical method of strain measurement in the split Hopkinson pressure bar", In: Wicks AL (ed.) Proceedings of IMAC-XIX: a conference on structural dynamics (vol. 2; Volume 4359 of Proceedings of SPIE). Bethel, CT: Society for Experimental Mechanics, pp.14711477, 2001.

[17] PÉREZ-MARTÍN, M.J., ERICE, B., GALVEZ, F. “On the loadingrate dependence of the Al 7017-T73 fracture-initiation toughness”, Procedia Mater Sci., v.3, pp.1026-1031, 2014.

[18] RODRIGUEZ PEREZ, J. Análisis y desarrollo de metodologías para la obtención de propiedades mecánicas de materiales a altas velocidades de deformación y a alta temperatura, Universidad Complutense de Madrid, 2002.

[19] RESNYANSKY, A. Study of influence of loading method on results of the split Hopkinson bar test. Structural Failure and Plasticity (IMPLAST), pp. 597-602, 2000.

[20] YANG, X, XIONG, X, YIN, Z., et al., "Interrupted test of advanced high strength steel with tensile split Hopkinson Bar method.”, Exp Mech., v.54, n.4, pp.641-652, 2014.

[21] NEMAT-NASSER, S, ISAACS, J., STARRETT, J. "Hopkinson techniques for dynamic recovery experiments”, Proc R Soc Lond A: Math Phys Eng Sci., v.435, pp. 371-391, 1991.

[22] DONGFANG, M, DANIAN, C, SHANXING, W, et al., "An interrupted tensile testing at high strain rates for pure copper bars", J Appl Phys., v.108, n.11, pp. 114902, 2010.

[23] WU, X., GORHAM, D. "Stress equilibrium in the split Hopkinson pressure bar test", J Phys IV, v.7(C3), pp.91-96, 1997.

[24] JIANG, F., VECCHIO, K.S. "Hopkinson Bar loaded fracture experimental technique: a critical review of dynamic fracture toughness tests”, Appl Mech Rev, v.62, n.6, pp.060802, 2009.

[25] GODUNOV, S.K., ROMENSKII, E.I. Elements of continuum mechanics and conservation laws, Novosibirsk: Springer Science+Business Media, 2003.

[26] LEZCANO R, ESSA, YES., PEREZ-CASTELLANOS, J. "Numerical analysis of interruption process of dynamic tensile tests using a Hopkinson bar”, J Phys IV, v.110, pp.565-570, 2003. 


\section{ORCID}

Roberto Alonso González Lezcano

https://orcid.org/0000-0002-6185-4929

Eduardo José López Fernández https://orcid.org/0000-0001-9103-3585

Sonia Cesteros García

https://orcid.org/0000-0002-8768-8279

Gastón Sanglier Contreras

https://orcid.org/0000-0002-8981-5622 